\title{
Signatures of long-lived spiral patterns: The color gradient trend
}

\section{Eric E. Martínez-García ${ }^{1}$ and Rosa Amelia González-Lópezlira ${ }^{2}$}

${ }^{1}$ Instituto de Astronomía, Universidad Nacional Autónoma de México, AP 70-264, Distrito Federal 04510, México; e-mail: martinez@astro.unam.mx. ${ }^{2}$ Centro de Radioastronomía y Astrofísica, UNAM, Campus Morelia, Michoacán, México; e-mail: r.gonzalez@crya.unam.mx

\begin{abstract}
Based both on observations and simulations, recent works propose that the speed of the spiral pattern in disk galaxies may decrease with increasing radius; the implications are that patterns are actually short-lived, and that the azimuthal color/age gradients across spiral arms predicted by density wave theory could not be produced. We, however, have consistently found such gradients, and measured spiral pattern speeds by comparing the observations with stellar population synthesis models (González \& Graham 1996; Martínez-García et al. 2009a,b; Martínez-García \& González-Lópezlira 2011). Here, we summarize our previous results in nonbarred and weakly barred spirals, together with six new, as yet unpublished, objects. On the other hand, we have indeed found a trend whereby pattern speeds at smaller radii are larger than expected from a model that assumes purely circular orbits (cf. Figure 1), likely due to the effect of spiral shocks on the orbits of newborn stars. The results suggest that spirals may behave as steady long-lived patterns.
\end{abstract}

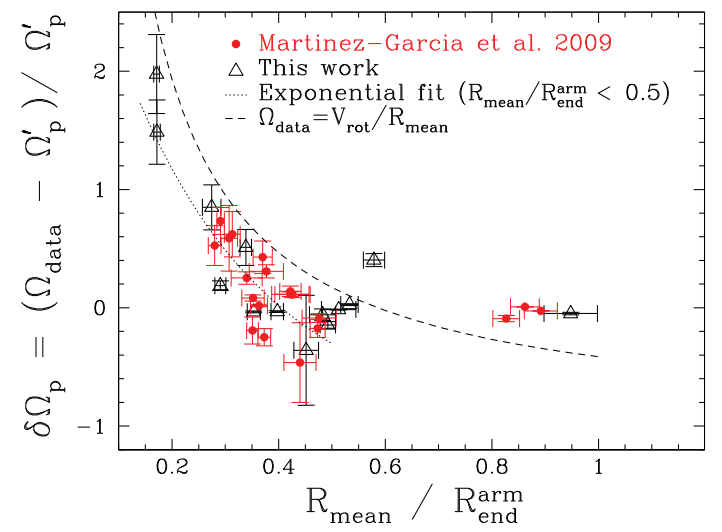

Figure 1. The quantity $\delta \Omega_{p}$ (cf. Martínez-García et al. 2009b) vs. $\frac{R_{\operatorname{mean}}}{R_{\text {end }}^{\text {arm }}}$ for 20 regions analyzed in Martínez-García et al. 2009a (red filled circles), and 14 unpublished regions (black empty triangles). The dotted line indicates an exponential fit for $\frac{R_{\text {mean }}}{R_{\text {end }}^{\text {ar }}}<0.5$. Long-dashed line: rotation
curve.

\section{References}

González, R. A. \& Graham, J. R. 1996, ApJ, 460, 651

Martínez-García, E. E., González-Lópezlira, R. A., \& Bruzual-A, G. 2009a, ApJ, 694, 512

Martínez-García, E. E., González-Lópezlira, R. A., \& Gómez, G. C. 2009b, ApJ, 707, 1650

Martínez-García, E. E., \& González-Lópezlira, R. A. 2011, ApJ, 734, 122 